\title{
A Quasi-Oppositional Heap-Based Optimization Technique for Power Flow Analysis by Considering Large Scale Photovoltaic Generator
}

\author{
Vedik Basetti ${ }^{1, *}$, Shriram S. Rangarajan ${ }^{1,2, *}$, Chandan Kumar Shiva ${ }^{1, *}$, Sumit Verma ${ }^{3}$, Randolph E. Collins ${ }^{2}$ \\ and Tomonobu Senjyu 4 (D) \\ 1 Department of Electrical and Electronics Engineering, SR University, Warangal 506371, India \\ 2 Department of Electrical and Computer Engineering, Clemson University, Clemson, SC 29634, USA; \\ collins@clemson.edu \\ 3 Department of Industrial and Management Engineering, IIT Kanpur, Kanpur 208016, India; sumitv@iitk.ac.in \\ 4 Department of Electrical and Electronics Engineering, University of the Ryukyus, Okinawa 903-0213, Japan; \\ b985542@tec.u-ryukyu.ac.jp \\ * Correspondence: b.vedik@sru.edu.in (V.B.); shriras@g.clemson.edu (S.S.R.); \\ chandan.kumar@sru.edu.in (C.K.S.)
}

check for updates

Citation: Basetti, V.; Rangarajan, S.S.; Kumar Shiva, C.; Verma, S.;

Collins, R.E.; Senjyu, T. A

Quasi-Oppositional Heap-Based Optimization Technique for Power Flow Analysis by Considering Large Scale Photovoltaic Generator. Energies 2021, 14, 5382. https://doi.org/ 10.3390/en14175382

Academic Editor: Nicu Bizon

Received: 25 July 2021

Accepted: 27 August 2021

Published: 30 August 2021

Publisher's Note: MDPI stays neutral with regard to jurisdictional claims in published maps and institutional affiliations.

Copyright: (c) 2021 by the authors. Licensee MDPI, Basel, Switzerland. This article is an open access article distributed under the terms and conditions of the Creative Commons Attribution (CC BY) license (https:/ / creativecommons.org/licenses/by/ $4.0 /)$.
Abstract: Load flow analysis is an essential tool for the reliable planning and operation of interconnected power systems. The constant increase in power demand, apart from the increased intermittency in power generation due to renewable energy sources without proportionate augmentation in transmission system infrastructure, has driven the power systems to function nearer to their limits. Though the power flow (PF) solution may exist in such circumstances, the traditional NewtonRaphson based PF techniques may fail due to computational difficulties owing to the singularity of the Jacobian Matrix during critical conditions and faces difficulties in solving ill-conditioned systems. To address these problems and to assess the impact of large-scale photovoltaic generator (PVG) integration in power systems on power flow studies, a derivative-free quasi-oppositional heap-based optimization (HBO) (QOHBO) technique is proposed in the present paper. In the proposed approach, the concept of quasi-oppositional learning is applied to $\mathrm{HBO}$ to enhance the convergence speed. The efficacy and effectiveness of the proposed QOHBO-PF technique are verified by applying it to the standard IEEE and ill-conditioned systems. The robustness of the algorithm is validated under the maximum loadability limits and high $\mathrm{R} / \mathrm{X}$ ratios, comparing the results with other well-known methods suggested in the literature. The results thus obtained show that the proposed QOHBO-PF technique has less computation time, further enhancement of reliability in the presence of PVG, and has the ability to provide multiple PF solutions that can be utilized for voltage stability analysis.

Keywords: load flow; differential evolution; photovoltaic generator; multiple solutions; high R/X ratio; and loadability limits

\section{Introduction}

Power flow analysis is a powerful and most widely utilized analytical tool to determine steady-state planning, operation, and energy management of the power system. The main objective of the power flow analysis is to determine the voltage phasors at all the buses by making use of the specified generation and load of the power system [1,2]. This load flow (LF) problem is solved by using various numerical computational techniques by utilizing the non-linear power injection models that are functions of bus voltage phasors and bus admittances [3]. In the literature [4], many techniques follow similar computation procedures to solve the LF problem. These techniques start with an initial guess to determine the real and reactive power injection mismatches at the nodal buses. Further, the problem variables are updated by utilizing power mismatches and the Jacobian matrix [1]. In [5], a fast load flow algorithm was suggested to solve both the transmission and distribution networks 
by considering a high impedance ratio of the systems. Similar to [5], the authors in [6] demonstrated a methodology that combined the fast decoupled load flow technique and complex per unit normalization method to deal with both transmission and distribution systems. The authors in [7] presented an algorithm that combined gradient descent and Newton-Raphson (NR) to solve power flow (PF) as an optimization problem. Here, in this approach, the inversion of the Jacobian matrix is eliminated using gradient descent steps. A modified traditional Gauss-Seidel method using a successive approximation technique was shown in [8]. In order to avoid the factorization of the inverse Jacobian matrix in the NR method to PF problem, an implicit formulation was suggested in [9]. Further, to enhance the robustness and L-stability of the approach, a backward Euler method was utilized in [9]. To solve uncertain PF analysis, an affine arithmetic method was explored in [10]. In [11], the application of two cubic methods, namely, Darvishi and Weerakoon-like approaches was examined to solve PF studies. An efficient and robust PF solver based on the Bulirsch-Stoer method was suggested in [12]. Among many computational techniques suggested in the literature [4], the NR technique and its variants are most widely used to solve the power flow method due to their reliable and very fast convergence characteristics. However, these techniques have various limitations such as (a) the tuning performance is highly dependent on the starting values (initialization) of the variables in the power flow problem; (b) it does not provide satisfactory results for ill-conditioned power systems, and it is incapable of providing solution during abnormal operating conditions; (c) the solution process diverges during critical operating conditions as Jacobian matrix becomes singular and during high $\mathrm{R} / \mathrm{X}$ ratio; and (d) it does not provide multiple solutions [13].

In the literature $[4,14]$, distinct efforts have been made to overcome the drawback of NR method in solving ill-conditioned power systems. The authors in [15] presented a robust four-stage PF solver. However, it was identified that even though this approach was quite effective for ill-conditioned system, it was not viable in comparison with NR for well-conditioned power systems. Similarly, in order to tackle ill-conditioned systems, a unified structure based on the Kronecker product that eliminates the truncation error is suggested in [16]. In [17], the Richardson extrapolation technique was presented not only to overcome the drawback of the conventional NR method in solving ill-conditioned systems but also during improper initialization in solving PF problems. A new technique, namely Predictor-Corrector NR was employed in [14] to PF problem to solve both well and ill-conditioned systems. It has been observed that using the mechanism of the PredictorCorrector, the convergence speed has also been improved to 2.4 from 2 in comparison with the conventional NR method. The authors in [18] examined the behavior of current injection formulation in solving ill-conditioned systems. It was found that the stability of the system had been improved in providing the solution with little computational savings. However, as stated in [14], this methodology was more appropriate in case of PQ buses (load buses) and displays a slow convergence rate in the case of PV buses (generation buses). In [19], a robust Levenbergy-Marquardt complex-valued algorithm was suggested in solving ill-conditioned problems. It was observed that this approach exhibited a bi-quadratic convergence rate in obtaining the solution to LF problems. However, the robustness of the algorithm has not been demonstrated with respect to the initial starting point [12]. Even though the methods discussed above can tackle ill-conditioned problems, these techniques do not provide multiple solutions, which is useful for stability analysis. Further, robustness with respect to the different starting points (initialization) has not been tested in most of the methods discussed above.

To overcome the drawbacks of the conventional NR method and to provide multiple solutions, evolutionary techniques have been suggested in the literature. In [13], an advanced constrained genetic algorithm (GA) was developed by considering the minimization of the total sum of squared mismatches (active power, reactive power, and voltage magnitude) as the objective function. A constrained GA load flow with a dynamic population method was presented in [20] to solve load flow under various operating conditions. A robust local search method was suggested in [21] to solve the LF problem. Further, the 
method has the ability to find the maximum loadability limit, P-V / P-Q curves, and multiple optimal solutions. However, this method was not tested on ill-conditioned systems. Similar to [13,21], the authors in [22] suggested an expert algorithm based on adaptive PSO (APSO) and chaotic particle swarm optimization (PSO) (CPSO) [23] to perform LF analysis. A decoupled power flow approach using the PSO technique was suggested in [1] by embedding flexible alternating current transmission system (FACTS) devices into the power system. Hybrid differential evolution (DE) and PSO-based LF technique was presented in [24] to overcome the limitations of conventional methods and PSO technique. However, these techniques require tuning of control parameters to obtain desired and accurate solution. Further, it is observed that the impact of the integration of PVGs with the power system on the power flow has not been studied in the literature discussed above.

The growing concern for global warming and depletion of non-renewable resources has necessitated integrating the photovoltaic (PV) generators (PVGs) with the power system [25]. In the literature discussed above, LF analysis has been performed by considering the synchronous generators where the power is generated at a specified voltage magnitude. However, modification in LF analysis modeling is required with the increased use of PVGs and their integration with the power grid [2]. Unlike conventional generators, these PVGs are stochastic in nature and, thus, the effect of these sources on the reliable operation of the transmission system has become a great concern to power system operators and planners. Therefore, it is essential to carry out extensive power flow analysis to understand the impact of these PVGs. Thus, various mathematical modeling has been developed in the literature to study the impact of the integration of PVGs by performing a number of computer simulation studies [26].

In [25], the authors presented a new model that integrates PVGs to the power grid using pulse width modulation (PWM) inverter. This PWM inverter is modeled in terms of its control parameters in the power flow studies such that the injection of active power, reactive power, and voltage with the system can be controlled directly. In [26], power flow analysis with a large-scale PV power plant connected to the grid was suggested. In contrast to the existing techniques, a unified approach that incorporates the state variables of the PV system along with the state variables of the power system during the iterative process was proposed. In [27], a steady-state three-phase grid-connected PV system was interfaced with the power system through power electronics (PE) converters. Therefore, modeling of the PV system consists of three parts, namely, DC, inverter, and AC parts. These three parts are interfaced by satisfying the power balance between the PE transformation and instantaneous power. However, as these methods are solved using the NR technique, they have the same limitations mentioned above when performing PF analysis. Further, these techniques have not been tested under maximum loadability limits and higher $\mathrm{R} / \mathrm{X}$ ratios.

Therefore, in order to overcome the disadvantages of conventional NR method and evolutionary methods, in the present paper, an algorithm that is free of tuning the algorithmic control parameters, namely the quasi-oppositional heap-based optimization (HBO) technique, is proposed to perform the power flow analysis. Further, the impact of considering PVGs in power flow analysis has also been studied.

The HBO [28] technique mimics the human behavior/interaction in an organization based on corporate rank hierarchy $(\mathrm{CORH})$. Here, the concept of CORH is mapped to the heap data structure, hence the name HBO. This method can provide a global optimal solution to large-scale problems. This technique has three stages, namely the communication between the co-workers and their immediate supervisor, the communication between the co-workers, and the employee's self-contribution to attain a global optimal solution. The main advantages of this technique are (i) it maintains a proper balance between exploration and exploitation, (ii) a self-adaptive parameter, which avoids the local optima and premature convergence is designed, and (iii) easy implementation. Additionally, to enhance the convergence rapidity and solution accuracy, an intelligent strategy, namely, the concept of quasi-oppositional based learning (QOBL) is incorporated into basic $\mathrm{HBO}$, thus resulting a novel QOHBO. 
The main contributions of the work are five-fold as follow.

1. A novel QOHBO technique is proposed to overcome the disadvantage of conventional power flow analysis.

2. The impact of large-scale PVG on LF analysis is studied.

3. The proposed method is able to provide multiple solutions that can be utilized for voltage stability analysis.

4. The efficiency of the proposed technique is tested by applying it to ill-conditioned systems.

5. Robustness of the algorithm is verified under maximum loadability limits and high $\mathrm{R} / \mathrm{X}$ ratios.

The rest of the paper is organized as follows. Problem formulation of load flow analysis is provided in Section 2. Section 3 explains the solution methodology using QOHBO. LF using QOHBO is explained in Section 4. Section 5 discusses the results obtained using the proposed method. Finally, Section 6 concludes the paper.

\section{Problem Formulation}

\subsection{Power Flow Problem}

The power flow problem models the non-linear equations, which relate the injected nodal power at system buses $\left(S_{i}^{s p}=P_{i}^{s p}+j Q_{i}^{s p}\right)$ with the bus nodal voltages $\left(V_{i} \angle \theta_{i}\right)$ and the system circuit parameters $\left(y_{i j}\right)$. Using this basic information, the main aim of LF or PF analysis is to find the voltage magnitudes at $(N-M)$ load or PQ buses and voltage phase angles at all the buses except the reference bus, i.e., $(N-1)$ buses [12,17]. In the process of determining these voltage phasors, the algorithm needs to satisfy both the reactive power limits of $M$ generators (at all the PV buses) and the power injections at all the buses. The complex power injected at any bus is expressed as:

$$
S_{i}=P_{i}+j Q_{i}=V_{i} \times I_{i}^{*}=V_{i} \times\left(\sum_{j=1}^{N} y_{i j} \times V_{j}\right)^{*}
$$

Hence, the active and reactive power injection at a bus can be calculated as follows:

$$
\begin{aligned}
& P_{i}=\sum_{j=1}^{N}\left|V_{i}\right|\left|V_{j}\right|\left|Y_{i j}\right| \cos \left(\theta_{i}-\theta_{j}-\delta_{i j}\right) \\
& Q_{i}=\sum_{j=1}^{N}\left|V_{i}\right|\left|V_{j}\right|\left|Y_{i j}\right| \sin \left(\theta_{i}-\theta_{j}-\delta_{i j}\right)
\end{aligned}
$$

In the present work, the voltage phasors at all the buses that determine the state of the power system are considered as state variables. Hence, the voltage angle at all buses except reference bus and voltage magnitude at PQ buses are formed as state vector of the PF problem, which needs to be determined. Thus, the PF state vector may be defined as:

$$
x=\left\{V_{P Q}, \theta_{P V}, \theta_{P Q}\right\}
$$

Thus, the active power and reactive power mismatches for a bus can be given as:

$$
\begin{gathered}
\Delta P_{i}=P_{i}^{s p}-\sum_{j=1}^{N}\left|V_{i}\right|\left|V_{j}\right|\left|Y_{i j}\right| \cos \left(\theta_{i}-\theta_{j}-\delta_{i j}\right)=P_{i}^{s p}-P_{i}^{c a l c} \\
\Delta Q_{i}=Q_{i}^{s p}-\sum_{j=1}^{N}\left|V_{i}\right|\left|V_{j}\right|\left|Y_{i j}\right| \sin \left(\theta_{i}-\theta_{j}-\delta_{i j}\right)=Q_{i}^{s p}-Q_{i}^{c a l c}
\end{gathered}
$$

Hence, the objective function of the LF analysis is to minimize the square of the difference between the specified and calculated power at a bus while maintaining the voltage phasor and reactive power constraints. This is mathematically represented as 


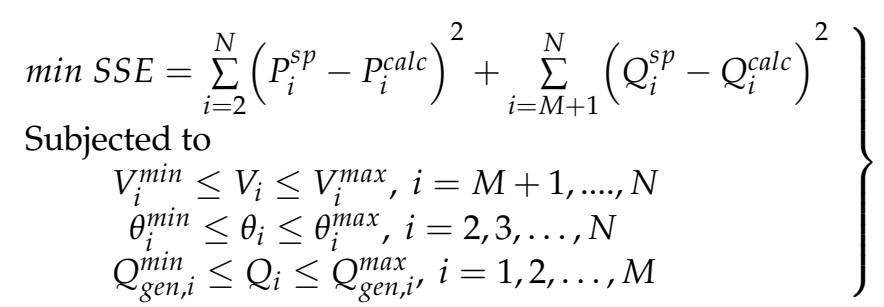

In the above equation, $M$ represents the number of generators; $N$ denotes the total number of buses in the power system, $V_{i}$ and $V_{j}$ signify the voltage magnitude at bus $i$ and $j$ respectively, $P_{i}^{c a l c}$ and $Q_{i}^{\text {calc }}$ represent the active and reactive power calculated at $i$ th bus, respectively, $y_{i j}$ denotes the admittance of the transmission line between $i$ th bus and $j$ th bus, $P_{i}^{s p}$ and $Q_{i}^{s p}$ indicate the active and reactive power specified at $i$ th bus, respectively, and subscripts "max" and "min" represent the corresponding maximum and minimum limits. Further, voltage magnitude at generator buses is fixed at the specified value [23].

\subsection{Power Flow Embedded with PVGs}

In the present work, a PVG is embedded in either a PV bus or a PQ bus of the power system under $V_{i n v}-\theta_{i n v}$ control mode. Further, the PVG is modeled as DC current source [25]. This DC current source injects $I_{P h}$ current that is proportionate to the incident solar radiation (insolation) up to the rated DC voltage $V_{D C}=V_{n o m-p h}$. Therefore, a fixed amount of active power, i.e., $P_{i n j}=V_{n o m-p h} \times I_{P h}$ is injected from the PVG if constant insolation persists. Thus, in the power flow analysis, this $P_{i n j}$ is considered as constant power injected at the embedded bus. Accordingly, the inverter bus is considered as an external bus with a predetermined inverter bus voltage phasors $V_{\text {inv }}$ and $\theta_{\text {inv }}$. This predetermined voltage phasors are obtained according to the method suggested in [25]. The modified active power mismatch equation with embedded PVG at a PV bus is given as follows.

$$
\begin{aligned}
& P_{i, p v}^{s p}=\left(P_{g e n-p v}+P_{i n j}-P_{l o a d-p v}\right) \\
& \Delta P_{P V}=P_{i, p v}^{s p}-P_{i, p v}^{c a l c} \\
& P_{i, p v}^{c a l c}=V_{i, p v} \times \sum_{j=1}^{N+i n v} V_{j}\left(G_{j-p v} \cos \theta_{j-p v}+B_{j-p v} \sin \theta_{j-p v}\right)
\end{aligned}
$$

In $V_{i n v}-\theta_{i n v}$ mode of operation, the modulation index, active power, and reactive power of the inverter are adjusted to retain the voltage phasor of the inverter bus at stated values. These values are predetermined when the PVG is embedded to a PV bus of the power system in the following way.

In general, the active power injected at a PV bus using the traditional generator and the voltage magnitude at this bus is maintained constant. Consequently, any additional power injected at this bus using any source requires modifying the voltage angle $\theta_{p v}$ only. Therefore, the relationship between the power injected by the additional source and the voltage angle $\theta_{p v}$ is expressed linearly as

$$
\theta_{p v}=a_{1} \times P_{i n j}+b_{1}
$$

where, $a_{1}$ and $b_{1}$ are the two constants that are computed offline. In order to obtain these values, the $P_{i n j}$ is randomly varied at the PV bus and corresponding $\theta_{p v}$ are computed by performing power flow analysis. After obtaining these values, a graph is plotted between $P_{i n j}$ and $\theta_{i n j}$. The slope of this line gives the values of $a_{1}$ and $b_{1}$. Further, the transfer of active and reactive powers from the inverter bus to the PV bus mainly depends on the voltage magnitude at the inverter bus and the phase angle difference between the PV and the inverter buses. Furthermore, the amount of active power injected depends on the $I_{p h}$, i.e., insolation. Accordingly, the relationship between inverter bus voltage $V_{i n v}$ and $\Delta I_{p h}$ and between $\theta_{i n v}$ and $\theta_{p v}$ are linearly expressed as follows. 


$$
\left.\begin{array}{c}
V_{i n v}=a_{2} \times \Delta I_{p h}+b_{2} \\
\partial=a_{3} \times \Delta I_{p h}+b_{3} \\
\theta_{i n v}=\theta_{p v}+\partial
\end{array}\right\}
$$

where, $a_{2}, a_{3}, b_{2}$, and $b_{3}$ are the constants that are computed off-line for various loading conditions for the considered power system according to [25]. After obtaining the values of $a_{1}, a_{2}, a_{3}, b_{1}, b_{2}$, and $b_{3}$ offline, the values of $V_{i n v}-\theta_{i n v}$ are calculated online for any value of $I_{\text {pho }}$ using (6) to (10).

\section{Solution Methodology Using QOHBO}

\subsection{Basic Heap-Based Optimization Technique}

In [28], the authors suggested a new algorithm known as a heap-based optimization technique based on the social behavior of human beings. This HBO technique was motivated based on the social interaction of human beings in an organization based on the concept of CORH. In order to map the concept of CORH, a heap-based data structure was utilized. An organization consists of a group of individual peoples who have arranged themselves in a hierarchy order, who works on a common objective to achieve the target. This CORH is similar to the structure of a tree. In this tree-like structure, the roots of a tree are considered the boss who is appointed at the top position and the staffs are considered as branches of a tree. Thus, the manager or boss is considered as a parent node and subordinates are considered as the children of the parent node. Here, each subordinate interacts with a fellow subordinate or the workers below him and follow the instructions of their boss to accomplish business-related contract in an optimal manner.

This entire process is fragmented into four different steps, which are explained in Appendix A Section.

\subsection{Quasi-Oppositional Based Learning}

The concept of opposition-based learning (OBL) was introduced by Tizhoosh in the year 2005. The main aim of this OBL concept is to enrich the accuracy of the solution and speed up the convergence rate to find a global solution. To perform QOBL, current candidate solution along with its quasi-opposite candidate solution is considered to attain an improved candidate solution. It has been proven by many researchers that a better opportunity to find a closer to the global minimum solution is by considering the opposite solution rather than selecting a randomly selected candidate solution $[29,30]$. The process to find a quasi-opposite candidate solution is elucidated by means of two key aspects, namely opposite vector, and quasi-oppositional vector.

These two aspects, i.e., opposite vector and quasi-oppositional vector, are explained in Appendix B section in detail.

\section{LF Using QOHBO Technique}

Similar to other meta-heuristic techniques, the proposed QOHBO is a populationbased method that starts with a group of search agents known as the initial population. Each search agent in the population denotes the candidate solution to the given problem. In the present work, the $\mathrm{QOHBO}$ is adopted to solve the LF problem. The complete solution methodology is explained as follows:

Step 1: Initialize the parameters of the algorithm and LF problem

In the present work, voltage phasors were considered as the state variables of the power system. To perform LF, these voltage phasors need to be initialized within the specified region. To obtain multiple LF solutions, the phase angles were generated randomly within the range of 15 and -180 degrees, and voltage magnitudes at different buses were initialized randomly between 0.3 p.u. and 1.1 p.u. The algorithm control parameters such as population size $(N)$, dimension size of variables $(D)$, and the maximum number of iterations (Max-iter) were initialized. The algorithmic control variable, i.e., the number of cycles was calculated as Max-iter/25. The size of the population and number of iterations were decided based upon the requirement of the user. 
Step 2: Initialize the population

Similar to all other evolutionary techniques, the QOHBO algorithm starts with the initialization of the population. The initial population is generated randomly according to uniform distribution within the search range. This initial population is known as the parent population.

$$
X_{i, 0}=l b+\operatorname{rand}(1, D) \cdot(u b-l b) \quad i=1,2, \ldots N P
$$

where $D$ is the dimension size of the problem. Here, the size of $D$ is twice the number of buses considered. For instance, the size of $D$ is 28 for IEEE 14-bus system. $l b$ and $u b$ refer to the lower and upper boundary of the variable. For example, the lower and upper boundary of the voltage magnitude is 0.4 and 1.1 , respectively.

Step 3: Compute the sum of the squares of mismatches

The fitness or value of each individual vector in the population is computed according to (5) by calculating the active and reactive power mismatches at each node. During this process, the voltage magnitude at each generator bus is kept fixed to the specified value.

Step 4: A ternary heap is considered to implement CORH. Even though heap is a tree-shaped data structure, it is efficiently implemented using an array [28].

Step 5: The fitness of each search agent is computed according to (5). If any search agent does not satisfy any constraints, then that infeasible search agent is penalized as discussed in Section 2.

Step 6: Now, perform quasi oppositional population for each search agent in current population.

Step 7: Select the best $N$ search agents among the quasi-oppositional population and current population. Then update the position of each search agent till the number of iterations reaches the maximum number of iterations.

The complete algorithm of QOHBO is given in Algorithm 1.

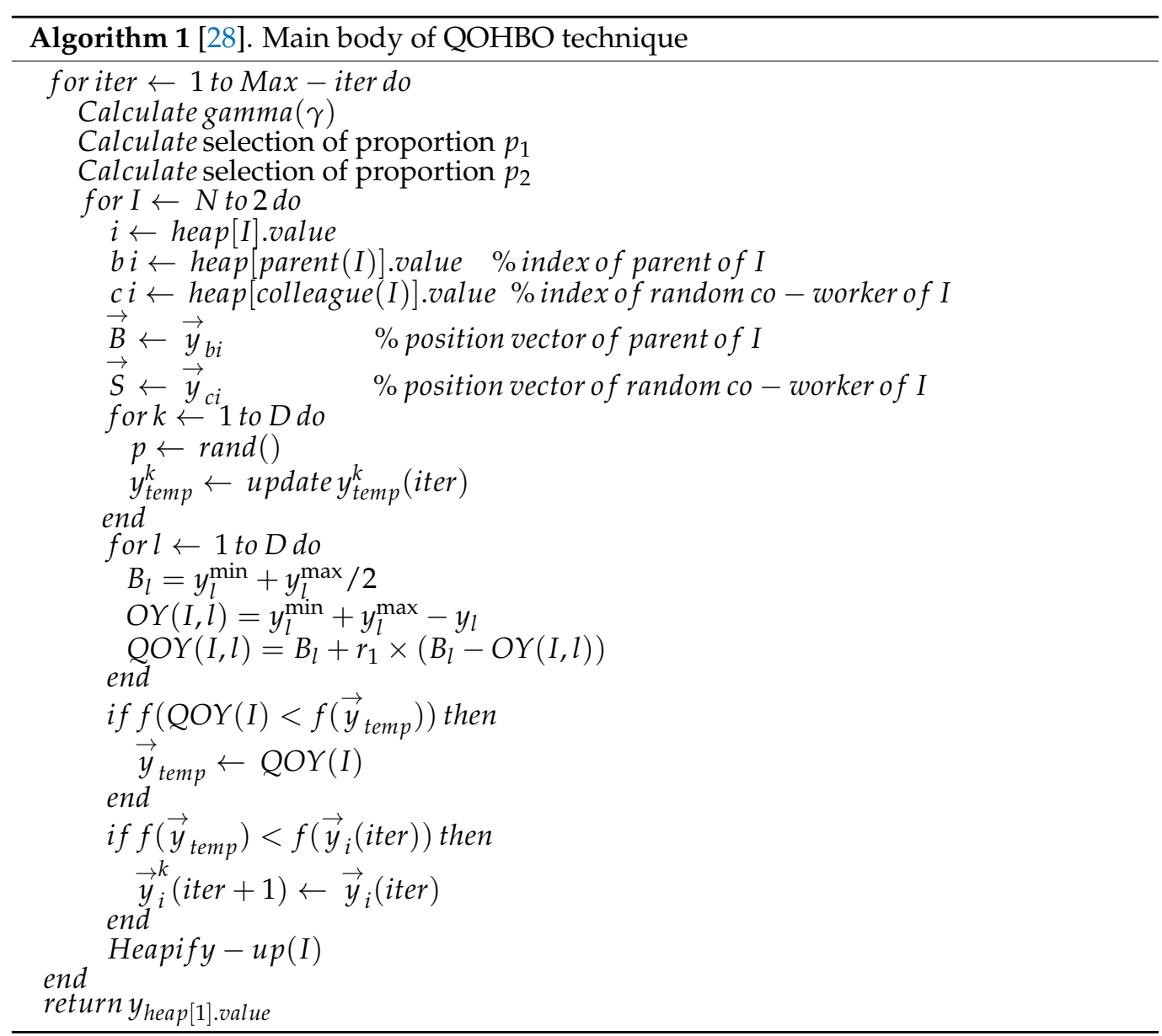


Step 8: Check convergence

If the iteration count reaches to maximum number of iterations, then print the results, else go to step 3.

\section{Results and Discussion}

The proposed QOHBO technique was applied to solve the load flow algorithm with an embedded PVG. To validate the efficacy and effectiveness, the proposed technique was tested on standard as well as ill-conditioned test systems [31]. The robustness of the algorithm was verified under maximum loadability limits and higher $R / X$ ratios. Further, the performance of the proposed technique was compared with the other techniques proposed in the literature. The proposed QOHBO-LFP with embedded PVG was implemented in MATLAB version 8.1.0.604 R2013a, and the program was run on Intel (R) Core ${ }^{\mathrm{TM}} \mathrm{i} 5$ (CPU) M480 @ 2.67 GHz processor with 4 GB RAM computer.

Unlike other evolutionary techniques suggested in the literature, QOHBO-LF depends only on two control parameters, namely population size and convergence criteria. Similar to other evolutionary techniques, the population size and convergence criteria depend on the size of the system under consideration. In the present work, a population size of 100 and the maximum number of iterations required to convergence was 10 times the size of the system considered. For example, the maximum number of iterations considered for IEEE 14-bus system is 140. The initial population of the algorithm is generated randomly between 0.3 p.u. and 1.1 p.u. for voltage magnitudes and between 15 deg. and $-180 \mathrm{deg}$. for voltage phase angles.

Case Study 1: Normal operating conditions

In this case study, LF analysis was performed using the QOHBO technique under normal operating conditions by considering with and without integration of PVG. The results thus obtained are tabulated in Tables 1 and 2 for 5-bus and IEEE 14-bus systems, respectively. Tables 1 and 2 consist of five columns. Columns 2 and 3 of Tables 1 and 2 represent the voltage magnitude and voltage angle obtained when PVG is not integrated with the system. For performing QOHBO-LF analysis with the integration of a PVG, a PVG having a generating capacity of $50 \mathrm{MW}$ and $25 \mathrm{kA}$ peak capacity at a rated voltage of $2 \mathrm{kV}$ has been considered at bus 3 (PV Bus) and bus 6 (PV Bus) of 5-bus and IEEE 14-bus systems, respectively. This PVG is integrated with 5-bus and IEEE 14-bus systems through a 60 MVA PWM inverter and a $1 \mathrm{kV} / 138 \mathrm{kV}$ transformer of 60 MVA having $6 \%$ impedance. After integrating the PVG at a specified bus, the values of $V_{i n v}-\theta_{i n v}$ are computed according to (6) and (10) for specified $I_{p h o}$. For obtaining the values of $V_{i n v}-\theta_{i n v}$ for any specified $I_{p h o}$, the constants in (9) and (10) are determined initially using the process explained in Section 2.2. The obtained values for both the systems are as follows.

\begin{tabular}{cccc}
\hline \multicolumn{2}{c}{ Five bus system: } & \multicolumn{2}{c}{ IEEE 14-bus system: } \\
\hline$a_{1}=0.0875$ & $b_{1}=-3.7031$ & $a_{1}=0.1331$ & $b_{1}=-14.4053$ \\
$a_{2}=-0.00184$ & $b_{2}=1.0691$ & $a_{2}=5 \times 10^{-5}$ & $b_{2}=1.6904$ \\
$a_{3}=-0.1034$ & $b_{3}=2.6334$ & $a_{3}=0.1002$ & $b_{3}=2.5049$ \\
\hline
\end{tabular}

Table 1. Load flow solutions for the five-bus system under normal operating conditions.

\begin{tabular}{ccccc}
\hline \multirow{2}{*}{ Bus No. } & \multicolumn{2}{c}{ Without Embedded PVG } & \multicolumn{2}{c}{ With Embedded PVG } \\
\cline { 2 - 5 } & $\begin{array}{c}\text { Voltage Magnitude } \\
\text { (P.U.) }\end{array}$ & $\begin{array}{c}\text { Voltage Angle } \\
\text { (rad) }\end{array}$ & $\begin{array}{c}\text { Voltage Magnitude } \\
\text { (P.U.) }\end{array}$ & $\begin{array}{c}\text { Voltage Angle } \\
\text { (rad) }\end{array}$ \\
\hline 1 & 1.0400 & 0.0000 & 1.0400 & 0.0000 \\
2 & 0.9614 & -0.1103 & 0.9621 & -0.0720 \\
3 & 1.0200 & -0.0648 & 1.0200 & 0.0026 \\
4 & 0.9203 & -0.1900 & 0.9203 & -0.1437 \\
5 & 0.9683 & -0.1075 & 0.9692 & -0.0779 \\
\hline
\end{tabular}


Table 2. Load flow solutions for IEEE 14-bus system under normal operating conditions.

\begin{tabular}{ccccc}
\hline \multirow{2}{*}{ Bus No. } & \multicolumn{2}{c}{ Without Embedded PVG } & \multicolumn{2}{c}{ With Embedded PVG } \\
\cline { 2 - 5 } & $\begin{array}{c}\text { Voltage Magnitude } \\
\text { (P.U.) }\end{array}$ & $\begin{array}{c}\text { Voltage Angle } \\
\text { (rad) }\end{array}$ & $\begin{array}{c}\text { Voltage Magnitude } \\
\text { (P.U.) }\end{array}$ & $\begin{array}{c}\text { Voltage Angle } \\
\text { (rad) }\end{array}$ \\
\hline 1 & 1.0600 & 0.0000 & 1.0600 & 0.0000 \\
2 & 1.0450 & -0.0870 & 1.0450 & -0.0620 \\
3 & 1.0100 & -0.2224 & 1.0100 & -0.1818 \\
4 & 1.0142 & -0.1790 & 1.0211 & -0.1267 \\
5 & 1.0172 & -0.1530 & 1.0262 & -0.1000 \\
6 & 1.0700 & -0.2516 & 1.0700 & -0.1033 \\
7 & 1.0503 & -0.2313 & 1.0500 & -0.1553 \\
8 & 1.0900 & -0.2313 & 1.0900 & -0.1553 \\
9 & 1.0337 & -0.2589 & 1.0284 & -0.1706 \\
10 & 1.0326 & -0.2625 & 1.0273 & -0.1635 \\
11 & 1.0475 & -0.2591 & 1.0437 & -0.1356 \\
12 & 1.0535 & -0.2665 & 1.0535 & -0.1223 \\
13 & 1.0471 & -0.2672 & 1.0455 & -0.1271 \\
14 & 1.0213 & -0.2804 & 1.0171 & -0.1695 \\
\hline
\end{tabular}

After obtaining the constants, QOHBO-LF analysis has been performed at a specified $I_{\text {pho }}$ of 16 . The results thus obtained using the proposed method are tabulated in columns 4 and 5 of Tables 1 and 2 for both 5-bus and IEEE 14-bus systems, respectively.

Case Study 2: Performance under loadability limit critical conditions

In this case study, the performance of QOHBO-LF has been tested by introducing the ill-conditioning in the test system by increasing the system loading. The results thus obtained using conventional Newton-Raphson load flow (NRLF) and proposed QOHBOLF techniques are shown in Table 3. Table 3 consists of four columns. The third column denotes the load multiplier, and the corresponding solution is given in the fourth column. Here, the load multiplier denotes the maximum value by which the system's active and reactive power loads have been manifolded. It may be noted here that the generator settings for the base case and during increased loading conditions were modified. Further, it is assumed here that the increased load demand is supplied by the slack bus. It can be observed from this table that the proposed QOHBO-LF method provides a solution to the problem even when the conventional NRLF fails. For example, NRLF fails to provide a solution to IEEE 14-bus system beyond the load multiplication factor of 3.6. This is because the Jacobian matrix of the NRLF method becomes singular for given loading conditions or the Jacobian matrix is near singular where the NRLF technique diverges. However, the proposed QOHBO-LF, which is a derivative-free technique, provides a solution up to the load multiplication factor of 3.98. This shows the robustness of the proposed method in comparison with the conventional NRLF technique. It is to be noted here that the situation at which the QOHBO-LF fails to provide the solution is the case at which there is no solution exists. The PV/QV curve obtained using the proposed QOHBO-LF technique for IEEE 14-bus system at buses 4 and 5 for various load multipliers has been shown in Figures 1 and 2, respectively. 
Table 3. Maximum load multiplier for loadability limit.

\begin{tabular}{|c|c|c|c|}
\hline Method & Test System & Load Multiplier & Solution \\
\hline NRLF & IEEE 5-bus System & 1.91 & 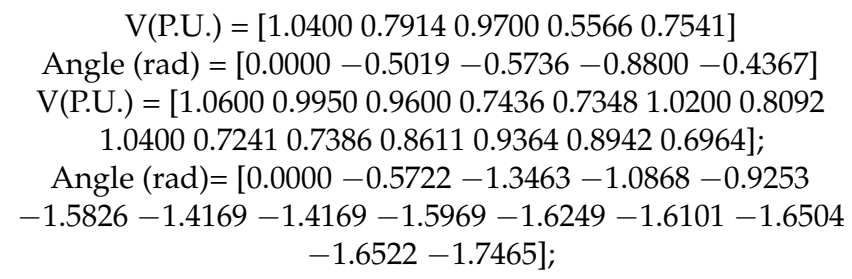 \\
\hline Proposed QOHBO & $\begin{array}{l}\text { IEEE 5-bus System } \\
\text { IEEE 14-Bus System }\end{array}$ & 3.98 & 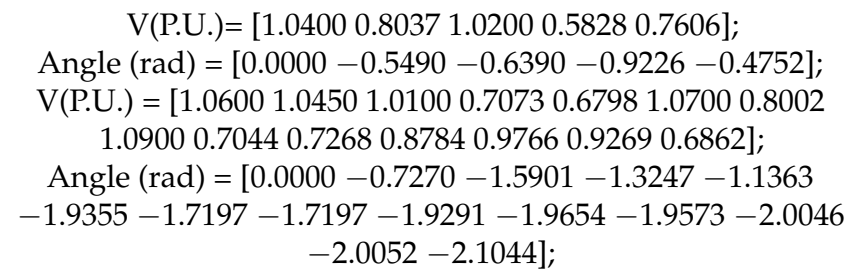 \\
\hline
\end{tabular}

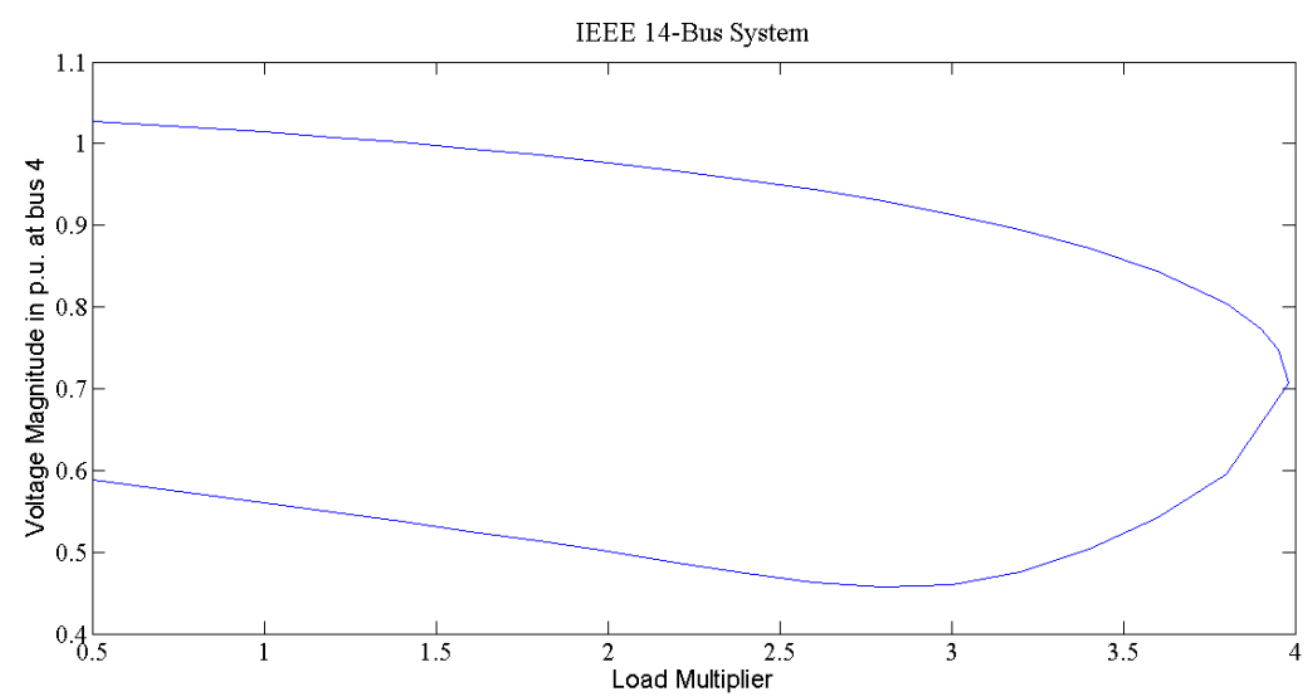

Figure 1. PV/PQ Curve for IEEE 14-bus system at bus 4.

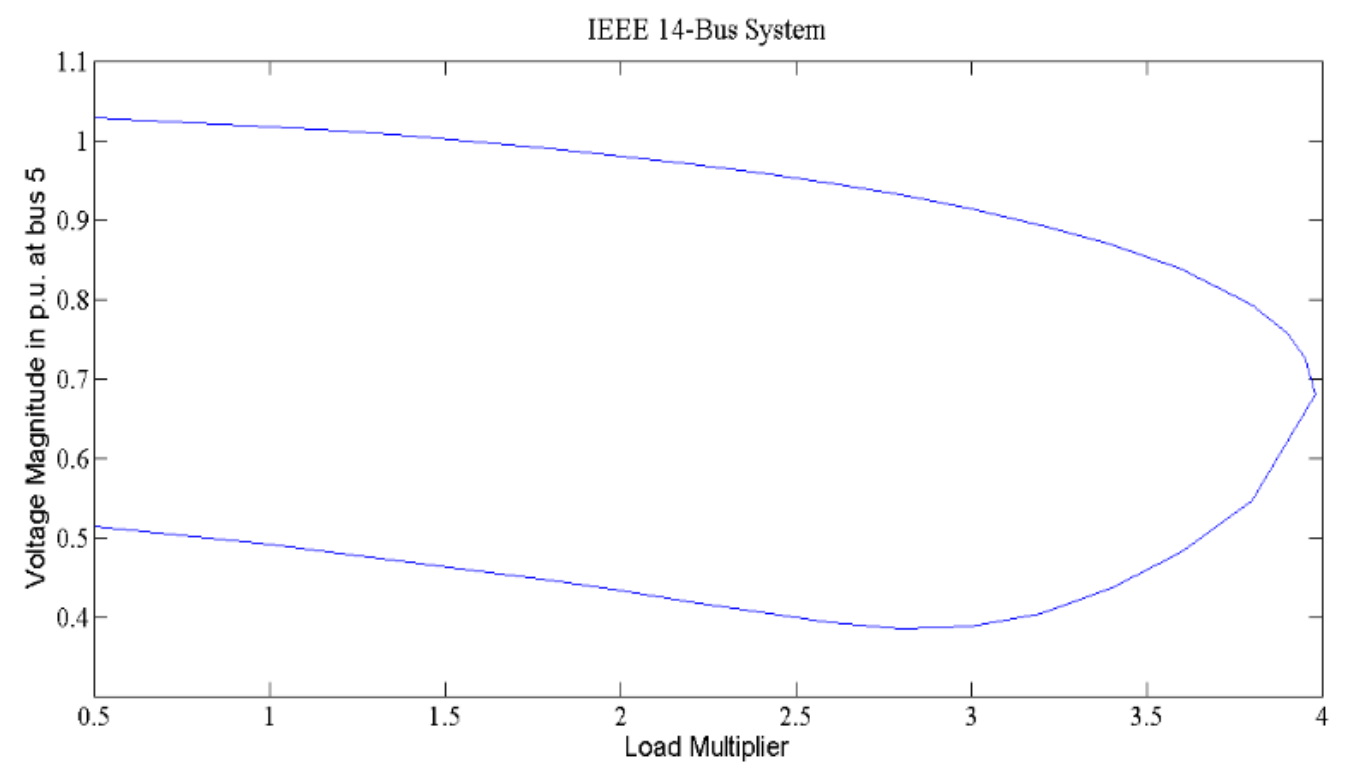

Figure 2. PV/PQ Curve for IEEE 14-bus system at bus 5. 
Case Study 3: Performance under high $\mathrm{R} / \mathrm{X}$ ratio critical conditions

In this case study, the performance of QOHBO-LF has been tested by introducing the illconditioning in the test system by either increasing the line resistance or by decreasing the line reactance. The results thus obtained using conventional NRLF and proposed QOHBOLF techniques by increasing the line resistance and by decreasing the line reactance are shown in Tables 4 and 5, respectively. Further, the results thus obtained using the proposed method was compared with NRLF with an optimal multiplier, local search, GA, and APSO techniques suggested in the literature. Tables 4 and 5 consist of three columns. Columns 2 and 3 denote the line resistance or line reactance multiplier for 5-bus and IEEE 14-bus test systems, respectively. Here, line resistance or line reactance multiplier denotes the maximum by which the system line resistances or line reactances have been multiplied by keeping baseload and generation constant. From Tables 4 and 5, it can be observed that the proposed method provides a solution to the problem even when other conventional and evolutionary techniques fail to converge. For example, the maximum line resistance multiplication factor at which NRLF, NRLF with an optimal multiplier, local search, GA, and APSO provide a solution for the IEEE 14-bus system are 4.47, 4.4225, 4.4288, 4.4371, and 4.4371 beyond which the techniques diverges, respectively. On the other hand, the proposed method even provides a solution with a line resistance multiplier of 4.5 for the IEEE 14-bus system. Further, it can be observed that this line resistance multiplier factor is increased to 4.9 when PVG is incorporated with the power system. Thus, with the integration of PVG into the system, the solution to the LF problem has been attained by satisfying the PF equations. Similarly, from Table 5, it can be observed that the proposed QOHBO-LF method provides a solution with a line reactance multiplication factor of 0.0418 where other conventional and evolutionary techniques diverge. Further, it has been observed, the line reactance multiplication factor can be further decreased from 0.0418 to 0.0406 to attain a solution when PVG is integrated into the power system, thus increasing the reliability of the power system.

Table 4. Line resistance multiplier for the critical condition.

\begin{tabular}{ccc}
\hline Solution Method & IEEE 5-Bus System & IEEE 14-Bus System \\
\hline NRLF & 4.9 & 4.47 \\
NRLF with Optimal multiplier [22] & - & 4.4225 \\
Local search [22] & - & 4.4288 \\
GA [22] & - & 4.4371 \\
APSO [22] & - & 4.4371 \\
Proposed QOHBO without PVG & 4.9 & 4.5 \\
Proposed QOHBO with PVG & 5.9 & 4.9 \\
\hline
\end{tabular}

Table 5. Line reactance multiplier for the critical condition.

\begin{tabular}{ccc}
\hline Solution Method & IEEE 5-Bus System & IEEE 14-Bus System \\
\hline NRLF & 0.012 & 0.043 \\
NRLF with Optimal multiplier [22] & - & 0.0479 \\
Local search [22] & - & 0.0428 \\
GA [22] & - & 0.0419 \\
APSO [22] & 0.001 & 0.0419 \\
Proposed QOHBO without PVG & 0.0001 & 0.0418 \\
Proposed QOHBO with PVG & & 0.0406 \\
\hline
\end{tabular}

Case Study 4: Performance under ill-conditioned system

In this case study, the reliability of the proposed QOHBO-LF in obtaining a solution for the ill-conditioned system has been tested. For this, LF analysis was performed on an ill-conditioned 13-bus system under normal conditions. The results thus obtained using the proposed method is given in Table 6. From Table 6, it has been observed that conventional methods such as NRLF, fast decoupled FL (FDLF), and Gauss-Seidel (GS) techniques have 
not converged [32]. On the other hand, the proposed method has been converged. The solution obtained for the 13-bus ill-conditioned system using the proposed method has been provided in Table 6 . This shows the reliability of the proposed method.

Table 6. Load flow solution for an ill-conditioned 13-bus system under normal conditions.

\begin{tabular}{|c|c|c|c|c|c|c|c|c|}
\hline \multirow[b]{2}{*}{ Bus No. } & \multicolumn{2}{|c|}{ Proposed QOHBO-LF } & \multicolumn{2}{|c|}{ NRLF [32] } & \multicolumn{2}{|c|}{ FDLF [32] } & \multicolumn{2}{|c|}{ GS [32] } \\
\hline & $\begin{array}{c}\text { Voltage } \\
\text { Magnitude } \\
\text { (P.U.) }\end{array}$ & $\begin{array}{c}\text { Voltage } \\
\text { Angle (rad) }\end{array}$ & $\begin{array}{c}\text { Voltage } \\
\text { Magnitude } \\
\text { (P.U.) }\end{array}$ & $\begin{array}{c}\text { Voltage } \\
\text { Angle (rad) }\end{array}$ & $\begin{array}{c}\text { Voltage } \\
\text { Magnitude } \\
\text { (P.U.) }\end{array}$ & $\begin{array}{c}\text { Voltage } \\
\text { Angle (rad) }\end{array}$ & $\begin{array}{c}\text { Voltage } \\
\text { Magnitude } \\
\text { (P.U.) }\end{array}$ & $\begin{array}{c}\text { Voltage } \\
\text { Angle (rad) }\end{array}$ \\
\hline 1 & 1.0000 & 0.0000 & & & & & & \\
\hline 2 & 0.9745 & 0.0409 & & & & & & \\
\hline 3 & 0.9426 & 0.0425 & & & & & & \\
\hline 4 & 1.0630 & 0.1588 & & & & & & \\
\hline 5 & 1.0442 & 0.0933 & & & & & & \\
\hline 6 & 1.0672 & 0.1433 & & & & & & \\
\hline 7 & 1.0177 & 0.2134 & $\mathrm{NC}$ & $\mathrm{NC}$ & $\mathrm{NC}$ & $\mathrm{NC}$ & $\mathrm{NC}$ & $\mathrm{NC}$ \\
\hline 8 & 0.9430 & 0.2525 & & & & & & \\
\hline 9 & 1.1000 & 0.1476 & & & & & & \\
\hline 10 & 1.1000 & 0.1438 & & & & & & \\
\hline 11 & 1.0000 & 0.0440 & & & & & & \\
\hline 12 & 1.0370 & 0.1723 & & & & & & \\
\hline 13 & 0.9693 & 0.0265 & & & & & & \\
\hline
\end{tabular}

NC: Not Converged.

Case Study 5: Multiple LF solutions

In this case study, one of the limitations of traditional techniques such as NRLF, FDLF, etc., that provides only a single LF solution is overcome by using the proposed QOHBO-LF technique. The proposed QOHBO-LF technique being a population-based evolutionary technique that has high exploration capability to find multiple solutions present in the entire search space. Some of the multiple LF solutions thus obtained using the proposed QOHBO-LF for IEEE 14-bus systems is shown in Table 7. This shows the effectiveness and reliability of the proposed method to provide multiple LF solutions.

Table 7. Multiple Load flow solutions for IEEE 14-bus system under normal conditions.

\begin{tabular}{ccccccc}
\hline & \multicolumn{2}{c}{ Solution 1 } & \multicolumn{2}{c}{ Solution 2 } & \multicolumn{2}{c}{ Solution 3 } \\
\cline { 2 - 7 } Bus No. & $\begin{array}{c}\text { Voltage } \\
\text { Magnitude } \\
\text { (P.U.) }\end{array}$ & $\begin{array}{c}\text { Voltage Angle } \\
\text { (rad) }\end{array}$ & $\begin{array}{c}\text { Voltage } \\
\text { Magnitude } \\
\text { (P.U.) }\end{array}$ & $\begin{array}{c}\text { Voltage Angle } \\
\text { (rad) }\end{array}$ & $\begin{array}{c}\text { Voltage } \\
\text { Magnitude } \\
\text { (P.U.) }\end{array}$ & $\begin{array}{c}\text { Voltage Angle } \\
\text { (rad) }\end{array}$ \\
\hline 1 & 1.0600 & 0.0000 & 1.0600 & 0.0000 & 1.0600 & 0.0000 \\
2 & 1.0450 & -0.2598 & 1.0450 & -0.0870 & 1.0450 & -2.4130 \\
3 & 1.0100 & -0.5856 & 1.0100 & -0.2224 & 1.0100 & -2.5765 \\
4 & 0.5607 & -0.4967 & 1.0142 & -0.1790 & 0.8014 & -2.4474 \\
5 & 0.4906 & -0.3076 & 1.0172 & -0.1530 & 0.6721 & -2.3380 \\
6 & 1.0700 & -3.2109 & 1.0700 & -0.2516 & 1.0700 & -2.5282 \\
7 & 0.5750 & -1.4133 & 1.0503 & -0.2313 & 0.9537 & -2.5039 \\
8 & 1.0900 & -1.4133 & 1.0900 & -0.2313 & 1.0900 & -2.5039 \\
9 & 0.4426 & -1.9847 & 1.0337 & -0.2589 & 0.9402 & -2.5299 \\
10 & 0.4554 & -2.3885 & 1.0326 & -0.2625 & 0.9548 & -2.5344 \\
11 & 0.7016 & -2.9596 & 1.0475 & -0.2591 & 1.0075 & -2.5326 \\
12 & 0.9857 & -3.2113 & 1.0535 & -0.2665 & 1.0465 & -2.5439 \\
13 & 0.9215 & -3.1658 & 1.0471 & -0.2672 & 1.0331 & -2.5429 \\
14 & 0.5129 & -2.7932 & 1.0213 & -0.2804 & 0.9613 & -2.5556 \\
\hline
\end{tabular}

Comparison of time with other techniques

The computational time taken to solve power flow analysis by various methods per iteration in seconds is tabulated in Table 8. It can be observed from this table that 
the proposed method provides a faster solution when compared to conventional and evolutionary techniques proposed in the literature. For example, the time taken per iteration by the proposed QOHBO-LF is $0.0105 \mathrm{~s}$ for 5-bus system, which is lesser when compared to other methods. This shows the computational efficiency of the proposed QOHBO-LF technique to solve power flow analysis.

Table 8. Computational time taken per iteration in seconds for various methods.

\begin{tabular}{cccc}
\hline Solution Method & 5-Bus System & IEEE 14-Bus System & $\begin{array}{c}\text { Ill-Conditioned } \\
\text { 13-Bus System }\end{array}$ \\
\hline NRLF & $0.0282 \mathrm{~s}$ & $0.0522 \mathrm{~s}$ & - \\
FDLF [1] & $0.0518 \mathrm{~s}$ & $0.0776 \mathrm{~s}$ & - \\
NRLF with Optimal & $0.0365 \mathrm{~s}$ & $0.0632 \mathrm{~s}$ & - \\
multiplier [22] & $0.0360 \mathrm{~s}$ & $0.585 \mathrm{~s}$ & - \\
Local search [22] & $0.0372 \mathrm{~s}$ & $0.0684 \mathrm{~s}$ & - \\
GA [22] & $0.0318 \mathrm{~s}$ & $0.0603 \mathrm{~s}$ & - \\
PSO method [1] & $0.0370 \mathrm{~s}$ & $0.0598 \mathrm{~s}$ & - \\
APSO [22] & $0.0498 \mathrm{~s}$ & $0.0601 \mathrm{~s}$ & $0.0101 \mathrm{~s}$ \\
PSO with update [1] & $0.0105 \mathrm{~s}$ & $0.0129 \mathrm{~s}$ & \\
Proposed QOHBO &
\end{tabular}

\section{Conclusions}

In the present work, the QOHBO technique with an embedded PVG is proposed to solve the LF problem. The efficiency, reliability, and robustness of the proposed method have been validated by applying it to standard and ill-conditioned systems under different conditions. The results obtained using the proposed method has been compared with other conventional and evolutionary techniques suggested in the literature. It has been observed that the proposed method delivers better performance when compared to other methods and provides a solution where other methods fail. The major findings of the proposed method are as follows.

- The proposed method has the ability to provide multiple solutions simultaneously. For instance, the proposed QOHBO has provided three solutions in a single run for the IEEE 14-bus system when compared to the single solution provided by the conventional NR method.

- The proposed method provides a solution to ill-conditioned systems where conventional techniques fail. For example, the proposed $\mathrm{QOHBO}$ has been able to provide a solution for ill-conditioned 13-bus system where conventional methods such as NRLF, FDLF, and GS failed to produce the solution.

- The proposed QOHBO method provides better performance under maximum loadability and higher $\mathrm{R} / \mathrm{X}$ ratios conditions. This performance is further enhanced with the integration of PVG. For instance, the line resistance multiplier for the critical conditions provided by QOHBO with PVG has been increased by 1, i.e., 5.9, when compared to NRLF and QOHBO, without PVG, is 4.9.

- The computational efficiency of the proposed method is higher when compared to other techniques. For example, the time taken by the proposed QOHBO to provide solution to IEEE 14 -bus system is $0.0129 \mathrm{~s}$ when compared to $0.0684 \mathrm{~s}$ provided by GA.

The proposed method is very useful and promising for solving practical problems that are non-linear and complex in nature. Further investigations can be carried out in applying the proposed method in solving LF problems by incorporating FACTS devices and reactive power planning.

Author Contributions: Conceptualization, C.K.S. and V.B.; methodology, C.K.S. and V.B.; software, S.V.; validation, S.S.R.; formal analysis, R.E.C.; investigation, R.E.C. and T.S.; writing-original draft preparation, C.K.S. and V.B.; writing-review and editing, S.S.R.; visualization, S.V. All authors have read and agreed to the published version of the manuscript. 
Funding: The author(s) received no specific funding for this work by any funding agency. This is the authors own research work.

Institutional Review Board Statement: Not applicable.

Informed Consent Statement: Not applicable.

Data Availability Statement: Data sharing is not applicable to this article.

Acknowledgments: The authors are grateful to their respective organizations for providing research opportunities and providing necessary resources towards completion of this paper.

Conflicts of Interest: The authors declared that they have no competing interest exist and have no conflict of interest among them.

\section{Appendix A}

The four different steps of the conventional $\mathrm{HBO}$ technique is explained as follows:

1. Modeling the $\mathrm{CORH}$

In modeling the $\mathrm{CORH}$, the concept of heap-based data structure is utilized. Here, a heap is considered as a complete tree that maps to complete the organization. Thus, the complete CORH is reflected as the population and each individual in the organization with the formal designation is considered as the search agent. Further, for min-heap, every parent fitness is either small or equal to its children. During implementation, the fitness of a search agent is conceived as the key of the corresponding node and the position of the agent is considered as the value of that node [28]. Thus, positions of the individuals in the organization are based on their fitness in the heap structure.

2. Modeling the interaction among the subordinates and their immediate supervisor/boss

In an organization, policies and rules are formed at the higher levels, and subordinates follow the order of their immediate supervisor/boss [28]. Therefore, the position of each search agent, i.e., subordinates, is updated regarding their immediate supervisor.

3. Modeling the interaction between the co-workers/colleagues

The individuals in the organization with the same designation are considered as colleagues or co-workers. These individuals interact among themselves to achieve the tasks assigned to them. Thus, it can be stated that each individual in the organization interacts with randomly selected other individuals of the same rank [28].

4. Modeling the individual contribution to the work

In this stage, the contribution of an individual search agent to the task is considered.

The detailed explanation of the $\mathrm{HBO}$ technique, including algorithms and flowchart, can be found in [28].

\section{Appendix B}

The two aspects, i.e., opposite vector and quasi-oppositional vector, are explained as follows.

\section{Appendix B.1. Opposite Vector}

As the name suggests, it generates a mirror solution to the current solution from the center of the search region. Therefore, the mirror image of $i$ th candidate solution of an $\mathrm{n}$-dimensional vector is generated according to (A1)

$$
O Y_{i}=y_{i}^{\min }+y_{i}^{\max }-y_{i}
$$

where $y_{i}^{\min }$ and $\mathrm{y}_{i}^{\max }$ denotes the minimum and maximum frontiers of parameters under study.

\section{Appendix B.2. Quasi-Oppositional Vector}

It is defined as the method of generating a random number between the opposite vector $\left(O Y_{i}\right)$ and the center of the search region $\left(\frac{y_{i}^{\min }+y_{i}^{\max }}{2}\right)$. It is mathematically defined as follows: 


$$
\mathrm{QOY}_{i}=\operatorname{rand}\left(\frac{y_{i}^{\min }+y_{i}^{\max }}{2}, y_{i}^{\min }+y_{i}^{\max }-y_{i}\right)
$$

After generating the quasi-opposition solution for each candidate solution of the given population, the fitness of quasi-opposition solutions is computed [29,30]. Then, based on the fitness value of the current population and quasi-opposition population, fittest $N$ individuals are selected $\{Y \cup Q O Y\}$ as the current population.

The pseudo-code for procurement of quasi oppositional number is presented below:

$$
\begin{gathered}
B=y^{\min }+y^{\max } / 2 \\
\text { if }(O Y<B) \\
\text { QOY }=B+r_{1} \times(O Y-B) ; \\
\text { else } \\
\text { QOY }=\text { OY }+r_{1} \times(B-O Y) ; \\
\text { end }
\end{gathered}
$$

where, $r_{1}$ denotes a random number generated between $(0,1)$.

\section{References}

1. Acharjee, P.; Goswami, S.K. A decoupled power flow algorithm using particle swarm optimization technique. Energy Convers. Manag. 2009, 50, 2351-2360. [CrossRef]

2. Kumar, P.; Singh, A.K. Load Flow Analysis with Wind Farms. In Handbook of Distributed Generation; Bansal, R., Ed.; Springer International Publishing: Cham, Switzerland, 2017; pp. 149-170. ISBN 978-3-319-51342-3.

3. Salomon, C.P.; Lambert-Torres, G.; Martins, H.G.; Ferreira, C.; Costa, C.I.A. Load flow computation via Particle Swarm Optimization. In Proceedings of the 2010 9th IEEE/IAS International Conference on Industry Applications-INDUSCON 2010, Sao Paulo, Brazil, 8-10 November 2010; IEEE: Piscataway, NJ, USA, 2010; pp. 1-6.

4. Karimi, M.; Shahriari, A.; Aghamohammadi, M.R.; Marzooghi, H.; Terzija, V. Application of Newton-based load flow methods for determining steady-state condition of well and ill-conditioned power systems: A review. Int. J. Electr. Power Energy Syst. 2019, 113, 298-309. [CrossRef]

5. Wang, Y.; Wu, H.; Xu, H.; Li, Q.; Liu, S. A general fast power flow algorithm for transmission and distribution networks. IEEE Access 2020, 8, 23284-23293. [CrossRef]

6. Portelinha, R.K.; Durce, C.C.; Tortelli, O.L.; Lourenço, E.M. Fast-decoupled power flow method for integrated analysis of transmission and distribution systems. Electr. Power Syst. Res. 2021, 196, 107215. [CrossRef]

7. Costilla-Enriquez, N.; Weng, Y.; Zhang, B. Combining Newton-Raphson and Stochastic Gradient Descent for Power Flow Analysis. IEEE Trans. Power Syst. 2021, 36, 514-517. [CrossRef]

8. Montoya, O.D.; Gil-González, W. On the numerical analysis based on successive approximations for power flow problems in AC distribution systems. Electr. Power Syst. Res. 2020, 187, 106454. [CrossRef]

9. Milano, F. Implicit Continuous Newton Method for Power Flow Analysis. IEEE Trans. Power Syst. 2019, 34, 3309-3311. [CrossRef]

10. Coletta, G.; Vaccaro, A.; Villacci, D. Fast and reliable uncertain power flow analysis by affine arithmetic. Electr. Power Syst. Res. 2019, 175, 105860. [CrossRef]

11. Albadi, M. Power Flow Analysis. Comput. Model. Eng. 2020, 1-15. [CrossRef]

12. Tostado-Véliz, M.; Kamel, S.; Jurado, F. A Robust Power Flow Algorithm Based on Bulirsch-Stoer Method. IEEE Trans. Power Syst. 2019, 34, 3081-3089. [CrossRef]

13. Wong, K.P.; Li, A.; Law, T.M.Y. Advanced constrained genetic algorithm load flow method. IEE Proc. Gener. Transm. Distrib. 1999, 146, 609-616. [CrossRef]

14. Tostado, M.; Kamel, S.; Jurado, F. Developed Newton-Raphson based Predictor-Corrector load flow approach with high convergence rate. Int. J. Electr. Power Energy Syst. 2019, 105, 785-792. [CrossRef]

15. Tostado-Veliz, M.; Alharbi, T.; Alrumayh, O.; Kamel, S.; Jurado, F. A Novel Power Flow Solution Paradigm for Well and Ill-Conditioned Cases. IEEE Access 2021, 9, 112425-112438. [CrossRef]

16. Oh, H. A Unified and Efficient Approach to Power Flow Analysis. Energies 2019, 12, 2425. [CrossRef]

17. Tostado-Véliz, M.; Kamel, S.; Jurado, F. Robust and efficient approach based on Richardson extrapolation for solving badly initialised/ill-conditioned power-flow problems. IET Gener. Transm. Distrib. 2019, 13, 3524-3533. [CrossRef]

18. Tostado-Véliz, M.; Kamel, S.; Jurado, F. Power flow solution of Ill-conditioned systems using current injection formulation: Analysis and a novel method. Int. J. Electr. Power Energy Syst. 2021, 127, 106669. [CrossRef]

19. Pires, R.; Mili, L.; Chagas, G. Robust complex-valued Levenberg-Marquardt algorithm as applied to power flow analysis. Int. J. Electr. Power Energy Syst. 2019, 113, 383-392. [CrossRef]

20. Wong, K.P.; Li, A.; Law, M.Y. Development of constrained-genetic-algorithm load-flow method. IEE Proc. Gener. Transm. Distrib. 1997, 144, 91. [CrossRef]

21. Acharjee, P.; Goswami, S.K. Robust load flow based on local search. Expert Syst. Appl. 2008, 35, 1400-1407. [CrossRef] 
22. Acharjee, P.; Goswami, S.K. Expert algorithm based on adaptive particle swarm optimization for power flow analysis. Expert Syst. Appl. 2009, 36, 5151-5156. [CrossRef]

23. Acharjee, P.; Goswami, S.K. Chaotic particle swarm optimization based robust load flow. Int. J. Electr. Power Energy Syst. 2010, 32, 141-146. [CrossRef]

24. Gnanambal, K.; Marimuthu, N.S.; Babulal, C.K. A Hybrid Differential Evolution Algorithm to Solve Power Flow Problem in rectangular coordinate. J. Electr. Syst. 2010, 6, 395-406. [CrossRef]

25. Shahnawaz Ahmed, S.; Mohsin, M. Analytical Determination of the Control Parameters for a Large Photovoltaic Generator Embedded in a Grid System. IEEE Trans. Sustain. Energy 2011, 2, 122-130. [CrossRef]

26. Juarez, R.T.; Fuerte-Esquivel, C.R.; Espinosa-Juarez, E.; Sandoval, U. Steady-State Model of Grid-Connected Photovoltaic Generation for Power Flow Analysis. IEEE Trans. Power Syst. 2018, 33, 5727-5737. [CrossRef]

27. Wang, Y.-B.; Wu, C.-S.; Liao, H.; Xu, H.-H. Steady-state model and power flow analysis of grid-connected photovoltaic power system. In Proceedings of the 2008 IEEE International Conference on Industrial Technology, Chengdu, China, 21-24 April 2008; IEEE: Piscataway, NJ, USA, 2008; pp. 1-6.

28. Askari, Q.; Saeed, M.; Younas, I. Heap-based optimizer inspired by corporate rank hierarchy for global optimization. Expert Syst. Appl. 2020, 161, 113702. [CrossRef]

29. Barisal, A.K.; Prusty, R.C. Large scale economic dispatch of power systems using oppositional invasive weed optimization. Appl. Soft Comput. J. 2015, 29, 122-137. [CrossRef]

30. Bhattacharjee, K.; Bhattacharya, A.; Dey, S.H.N. Oppositional Real Coded Chemical Reaction Optimization for different economic dispatch problems. Int. J. Electr. Power Energy Syst. 2014, 55, 378-391. [CrossRef]

31. Tiew On, T. Development of Hybrid Constrained Genetic Algorithm and Particle Swarm Optimisation Algorithm for Load Flow; The Hong Kong Polytechnic University: Hong Kong, China, 2007.

32. Mallick, S.; Rajan, D.V.; Thakur, S.S.; Acharjee, P.; Ghoshal, S.P. Development of a new algorithm for power flow analysis. Int. J. Electr. Power Energy Syst. 2011, 33, 1479-1488. [CrossRef] 